\title{
Logbuch Mathematik
}

Thilo Kuessner

Die Mengenlehre? Ganz einfach! Wenn in einem Raum drei sind und vier rausgehen, muss einer wieder rein, damit keiner drin ist.

Lothar Späth ${ }^{1}$

Eine besondere Sumpfblüte der Bildungsreform. Gerhard Mayer-Vorfelder

Sparen heißt, Geld, das man hat, nicht auszugeben. Bei uns geht es aber darum, Geld, das wir nicht haben, nicht auszugeben, und das nennt man Realismus. Ich darf dies vielleicht in der Sprache der Mengenlehre erläutern: Wenn man aus eine Kasse, in der 100 Mark drin sind, 300 Mark rausnimmt, muss man erst wieder 200 Mark reintun, damit nichts mehr drin ist.

Manfred Rommel

\section{Macht Mengenlehre krank?}

Der SPIEGEL hat eine wöchentliche Kolumne „Zeitreise der SPIEGEL vor 50 Jahren“, in der jeweils die interessantesten Artikel des 50 Jahre zurückliegenden Heftes aus heutiger Sicht kommentiert werden. Im Heft 12/2020 wurde also an Heft 12/1970 erinnert. Das hatte in der Rubrik „Schulen“ die damals an nordrhein-westfälischen Grundschulen neu erprobten Lehrpläne vorgestellt.

Die „starke bäuerlich-handwerklich-kaufmännische Ausrichtung“ des bisherigen Rechenunterrichts genügt nach jüngsten pädagogischen Forschungen nicht mehr den modernen Anforderungen. Denn das Rechnen mit Zahlen, das vor allem schematisches Denken erfordert, wird im Berufsleben mehr und mehr von Maschinen erledigt. Die neue Mathematik, die auf der sogenannten Mengenlehre basiert, soll dagegen logisches und analytisches Denken fördern. Die Erstkläßler beispielsweise müssen eine Menge von eckigen und runden, roten und blauen, großen und kleinen Figuren nach Form, Farbe und Größe sortieren. Durch diese Methode, die von Schuljahr zu Schuljahr anspruchsvoller wird, soll bei den Schülern - so die nordrhein-westfälischen Richtlinien - die „Fähigkeit des Ordnens [...] des Erfassens von Strukturen entwickelt werden“.

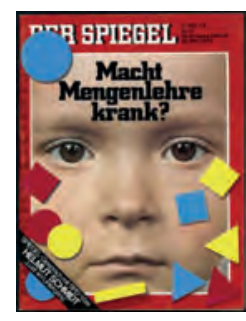

So also in Heft 12/1970. Ziemlich genau vier Jahre danach schaffte es die Mengenlehre dann sogar auf das Titelbild von Heft 13/1974. Doch der Enthusiasmus war dem Entsetzen gewichen.

Mengenlehre: „3+5 = 5+3“ war der Leitartikel des Heftes überschrieben. Es ging um Proteste und Prozesse gegen die Mengenlehre an Grundschulen.
Geschweifte Klammern und Ellipsen, in die immer neue und immer andere Mengen geschrieben oder gezeichnet werden, füllen viele Hefte. Väter und Mütter, die pflichtbewußt den Bestseller Eltern lernen die neue Mathematik oder ein anderes der fünf Dutzend Elternbücher gelesen oder einen Kurs an der Volkshochschule besucht haben, sind ihren Kindern wenigstens in der Erkenntnis voraus, daß es Mengen in Unmengen gibt: unter anderem Grund-, Teil-, Vereinigungs-, Ergänzungs-, Schnitt-, Unterschieds-, Null-, Verbindungs-, Rest-, ProduktLösungsmengen.

Aber selbst allabendlich strebend bemühten Eltern fällt es oft schwer, mit ihren Sprößlingen mitzuhalten oder ihnen zu helfen, wenn sich die Begriffe verwirren.

Von Mächtigkeit reden Achtjährige und meinen nicht Könige oder Kanzler, sondern Mengen von Haselnüssen und Rosinen. Und wenn sie sagen, irgend etwas sei irgend etwas anderem „eineindeutig“ zuzuordnen, dann stottern sie nicht, sondern sind stolz darauf, daß sie dem Vater auch dann überlegen sind, wenn er Abitur und Doktortitel besitzt. Laut Mengenlehre-Gegner Hans Stahl (Stuttgart) „sehen die Kinder früh, zu früh, ihre Eltern hilflos und unwissend. Damit schwindet die Achtung, die Kinder können nicht mehr ihre Eltern fragen, deren Vorbild verblaßt".

Klare Kampflinien gab es immerhin zwischen den akademischen Disziplinen:

Während Ärzte, Ärztekammern und -verbände vorerst nur vereinzelt gegen die Mengenlehre kämpfen, hat sich eine andere akademische Sparte fast vollzählig mit den empörten Eltern verbündet. Es sind die Universitätsprofessoren für Mathematik, die von der Art, wie Mengenlehre derzeit an deutschen Grundschulen betrieben wird, nicht viel mehr als nichts halten. 


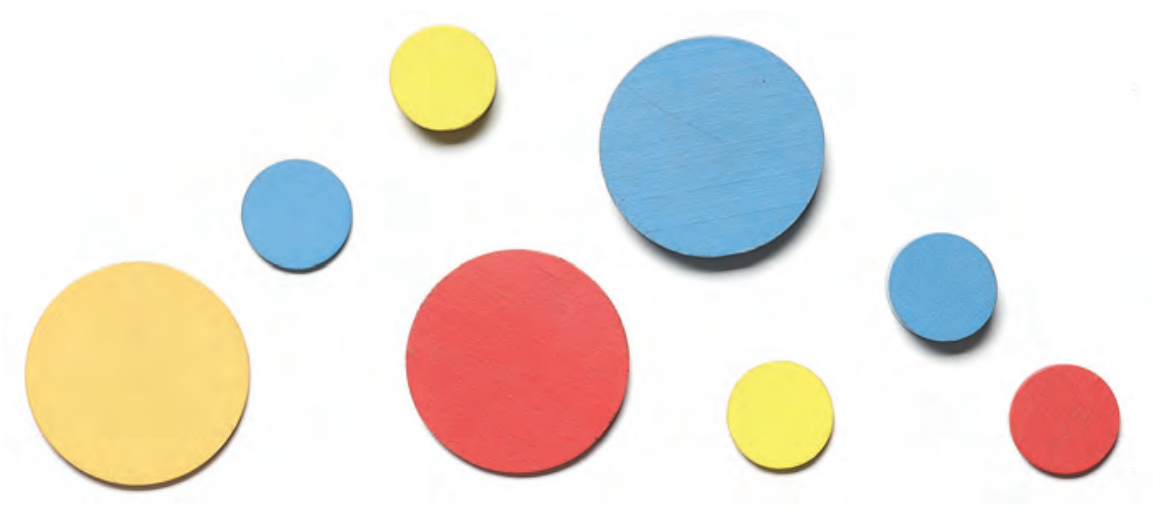

Mengenlehre sei zwar, argumentiert die „Deutsche Vereinigung für mathematische Logik", eine „wichtige mathematische Disziplin“, aber für die Schule kaum geeignet. Dort könne es allenfalls eine "Gebrauchsmengenlehre“ geben, die „eher eine Sprache als ein eigener mathematischer Stoff" sei und deshalb im Zusammenhang mit anderen Stoffen „allmählich und zwanglos eingeführt werden“ solle.

Die Gegenpartei bilden, nahezu ebenso geschlossen, die Professoren für Didaktik der Mathematik, die an den Pädagogischen Hochschulen tätig sind. Sie sind auch als Schulbuch-Autoren bemüht, der Grundschule das neue Gebiet zu eröffnen, um Anschluß an die weiterführenden Schulen zu halten.

Es lohnt, den langen Artikel ${ }^{2}$ in Gänze zu lesen.

\section{Leserkommentare}

In den scienceblogs haben wir aus Anlaß des 50-jährigen Jubiläums diejenigen Leser, die damals zur Schule gingen, gebeten, ihre Erfahrungen aus heutiger Perspektive zu schildern. ${ }^{3}$ Die Ergebnisse fand ich recht überraschend. Einige Antworten:

rinja: Ich profitiere in meinem Job als Softwareentwickler wunderbar von der in der Grundschule erlernten Mengenlehre. Ich habe mich oft gefragt, warum meine jüngeren Kollegen mit SQL (Sprache zur Datenabfrage) so gar nichts anfangen können. Im Gegensatz dazu ist für mich das Konzipieren von Abfragen, und somit von Teil-, Schnitt-, Unter- und sonstigen Mengen etc. quasi intuitiv möglich. Ich kann mich auch erinnern, dass mir der damalige Matheunterricht mit seinen bunten Plättchen und den zugehörigen Schablonen zum Zeichnen der Formen sehr viel Spass gemacht hat. Sicher mehr Spass als meinem Sohn das dauernde Rechnen macht.

PhD, DiplPhys: Ich habe damals Mengenlehre in der ersten Klasse gehabt, und es als unheimlich nützlich empfunden. Jetzt lehre ich an einer FH und würde mir wünschen, wir hätten den Mengenlehre-Unterricht beibehalten. Mir wurde damals eine Grundlage gelegt, wie man Probleme strukturiert, sortiert, in Teilaufgaben zerlegt, wie man mit Mathematik sprechen kann. Der Aufbau dieser Grundlagen ist heute mein Tagesgeschäft mit jungen Erwachsenen.

Philosoph: Der Wegfall der Mengenlehre war ein Kardinalfehler. Die Struktur der Computer, das Verständnis für Logikbausteine wird durch die Mengenlehre sehr erleichtert. Warum gibt es so wenig Systemanalytiker?

Inalidu: Ich habe Mengenlehre zwar als Grundschüler lustig gefunden, vor allem die vielen bunten Bildchen, die man malen konnte. Allerdings habe ich damit nicht Rechnen gelernt, sondern malen. Bis heute kann ich nicht Kopfrechnen, das Einmaleins haben wir nicht gelernt, alle Grundrechenarten fehlen weitgehend. Zum Glück konnte meine Generation Mathe im Abi noch abwählen. Vielleicht wäre es zusätzlich zu klassischer Mathe ganz gut gewesen. So war es nicht wirklich gut für „Mathe-minderbegabte“ Schüler. Wie immer kommt es eben sehr auf die konkrete Ausgestaltung an. Soweit ich mich erinnere, hat bei uns die Verknüpfung mit den "richtigen“ Zahlen einfach gefehlt, so dass für mich Mengenlehre zwar auch einfach und intuitiv war, aber eben nicht als „Mathematik“ erkennbar. Das Umsetzen der netten gemalten Dreicke oder dicken und dünnen Scheiben (für ein Kind ganz konkrete Dinge) auf abstrakte Zahlen hat irgendwie nicht funktioniert. Da ich kein Mathe-Talent habe, habe ich die durch mangelnde Übung fehlenden Grundlagen kaum mehr nachgeholt. Zahlen sind für mich immer eher fremd geblieben. Auch bei tollen Methoden wird es halt immer Kinder geben, die damit nicht so gut klarkommen. (Das gilt natürlich für alle Methoden).

Thomas: Es ist wie immer: Mathematik wird im "Volksmund“ mit „Rechnen“, mit dem Verknüpfen von Zahlen, gleichgesetzt. Als Kind der 7oer wurde ich auch mit der Mengenlehre in der Grundschule "belästigt“ ... und fand sie toll. Ich hatte nicht wirklich Probleme, die Konzepte dahinter zu verstehen (auch wenn ich als Kind noch nicht begriff, was das wohl alles soll. Aber man lernt es eben), aber mit einiger Verzögerung hat es mir geholfen, viele weiter Konzepte zu verstehen ..., so Sachen wie „Formale Sprachen“, „Entscheidbarkeit“, „Komplexitätstheorie“ ..., alles, was ein Informatikdiplomstudium der 8oer/goer Jahre ausmachte. Ich war schon ziemlich erschüttert, als meine Tochter dieses, in meinen Augen wichtige Rüstzeug der Mathematik in den frühen zoooern nicht mehr in der Grundschule gelehrt bekam. 
Peggy Sylopp: Mengenlehre war ein super Fach, ich habe die Legomaplättchen geliebt! Ich erinnere mich gut daran, wie ich immer wieder über den Rand der Plättchen strich, um haptisch in "glatt" oder "gezackt" zu unterteilen. Das Ordnen und Sortieren der Plättchen hat mir später im Informatikstudium beim Verstehen von Sortier-Algorithmen geholfen. Ich habe vor kurzem Mathe-Grundkurs in der 11. Klasse unterrichtet und musste feststellen, dass die meisten Schüler ${ }^{*}$ innen keine Vorstellung von Mengen haben. Das fällt ganz schön auf die Füße, wenn es um die Grundlagen von Funktionen, sprich Abbildungen von Mengen, geht. Sehr schade, dass Mengenlehre abgeschafft wurde.

user unknown: Ich hatte es in den 7oern in der Grundschule. Erst viel später hörte ich, dass es als Irrweg verspottet wurde, konnte mich dem aber nicht anschließen. Auch ich habe es beim Lernen von SQL gleich wiedererkannt. Geschadet hat es natürlich nicht (wie sollte es?), aber ich weiß natürlich nicht, was es verdrängt hat, was ich sonst gelernt hätte.

Stephanie: Also ich habe 1986 in Hessen nahe Frankfurt noch Mengenlehre gehabt und es war für mich sehr intuitiv und schön zu lernen. Heute bin ich echt froh, es gelernt zu haben, so dass mir vieles beim Programmieren leicht fällt.

\section{Geschichte eines Scheiterns}

Es ist natürlich möglich, dass die Leserschaft der scienceblogs keine repräsentative Stichprobe der Grundgesamtheit ist; die Antworten scheinen ja auch überwiegend von Lesern mit einem Bezug zu Informatik und Programmiersprachen $\mathrm{zu}$ stammen.

Vielleicht wäre das mal ein Thema für erziehungswissenschaftliche Master- oder Doktorarbeiten: herauszufinden, wie damalige Schüler ihren Mathematikunterricht heute einschätzen und inwieweit - subjektiv in der Eigensicht oder möglichst objektiv in der Außensicht - die erlernten und eventuell nicht erlernten Fähigkeiten ihnen genutzt oder auch geschadet haben.

Besser als der reale Unterricht lassen sich die theoretischen und unterrichtskonzeptionellen Hintergründe der damaligen Reform anhand von Quellen, also erziehungswissenschaftlicher und psychologischer Fachliteratur und vor allem damaliger Schulbücher und Lehrermaterialien rekonstruieren. Eine vor zwei Jahren an der Universität Hildesheim verfaßte Dissertation Die „Mengenlehre im Anfangsunterricht - Historische Darstellung einer gescheiterten Unterrichtsreform in der Bundesrepublik Deutschland" leistet dies durch Vergleich dreier damaliger Lehrwerke. Die Autorin Tanja Hamann ${ }^{4}$ konstatiert, die Mengenlehre sei als gescheitert anzusehen, weil „die Nähe der Umsetzung von Reformkonzepten zu den Ideen und Zielen, die ihren ursprünglichen Ausgang markieren“, nicht gegeben sei, sie „vielmehr durch eine Fülle an Anpassungen und Verkürzungen, auf den verschiedenen Ebenen“ gekennzeichnet gewesen wäre. Dennoch sei nicht alles, was im Zuge der Reform neu war, aus dem Unterricht verschwunden. Die Geometrie gehöre erst seit den 1970ern zum festen Kanon der Grundschule. Das Nachdenken darüber, welche Methode am geeignetsten ist, habe sich etabliert,
Lernspiele bei Schulanfängern und Gruppenarbeit würden so wenig in Frage gestellt wie offener Unterricht allgemein. Schulbücher seien weiterhin bunt und enthielten viele Bilder, Begriffe sollten nicht vorgegeben, sondern erarbeitet werden. Die Bezeichnung des Faches als „Mathematik" sei geblieben, eine Rückkehr zum alten Fach „Rechnen“ habe nie ernsthaft zur Debatte gestanden.

Interessant ist auch, was man in der Arbeit zur Geschichte der Reform liest:

Ende 1973 und damit nach dem ersten abgeschlossenen Schuljahr, in dem die Reform in der Praxis implementiert worden war, ergriff eine wohl beispielslose Protestwelle gegen die Neue Mathematik die Bundesrepublik. Dabei war die Reform des Mathematikunterrichts an den weiterführenden Schulen kein Thema, der Unmut richtete sich allein gegen die „Mengenlehre“ in der Grundschule. [...] Ein Jahr später waren sämtliche Massenmedien auf eine hysterische Debatte aufgesprungen, an der praktisch die gesamte Öffentlichkeit der Bundesrepublik Anteil nahm. Einen Eindruck von der Situation vermittelt Der Spiegel vom 25. März 1974, der der Reform unter der bemerkenswerten Schlagzeile „Macht Mengenlehre krank?“ seine Titelseite widmete.

[...]

Neben den Anschaffungskosten für Material und der häufig geäußerten Sorge, eine Kürzung der Arithmetik zugunsten der neuen Inhalte würde unweigerlich zu schwächeren Rechenleistungen führen, war ein Argument, vermutlich ausgelöst durch fehlendes eigenes Verständnis der neuen mathematischen Inhalte, dass Eltern ihren Kindern nicht mehr bei den Hausaufgaben helfen konnten. Ob dies überhaupt in großem Umfang nötig war, bleibt indes unklar. In dem im Spiegel wiedergegebenen Zitat eines „Mengenlehre-Gegner[s], die Kinder [sehen] früh, zu früh, ihre Eltern hilflos und unwissend. Damit schwindet die Achtung, die Kinder können nicht mehr ihre Eltern fragen, deren Vorbild verblaßt" kommen jedoch tieferliegende gesellschaftliche Überzeugungen zum Ausdruck, die sich gegen die emanzipatorischen Ziele der Reform richten und in der öffentlichkeitswirksamen Verteufelung der „Mengenlehre“ ein eher zufälliges Ventil finden. Wie hoch in jedem Fall das Informationsbedürfnis über das, was im Mathematikunterricht geschah, in der Elternschaft war, belegen der Erfolg speziell für Eltern geschriebener Bücher über die Neue Mathematik, die Tatsache dass entsprechende Volkshochschulkurse Anklang fanden und auch die 1974 eigens zum Thema vom Niedersächsischen Kultusministerium herausgegebenen Hilfen zur Durchführung von Elternabenden.

[...]

Der heute so absurd anmutende Spiegel-Titel war weder reine Ironie noch bewußte Provokation, sondern gab wieder, was einige Ärzte tatsächlich öf- 


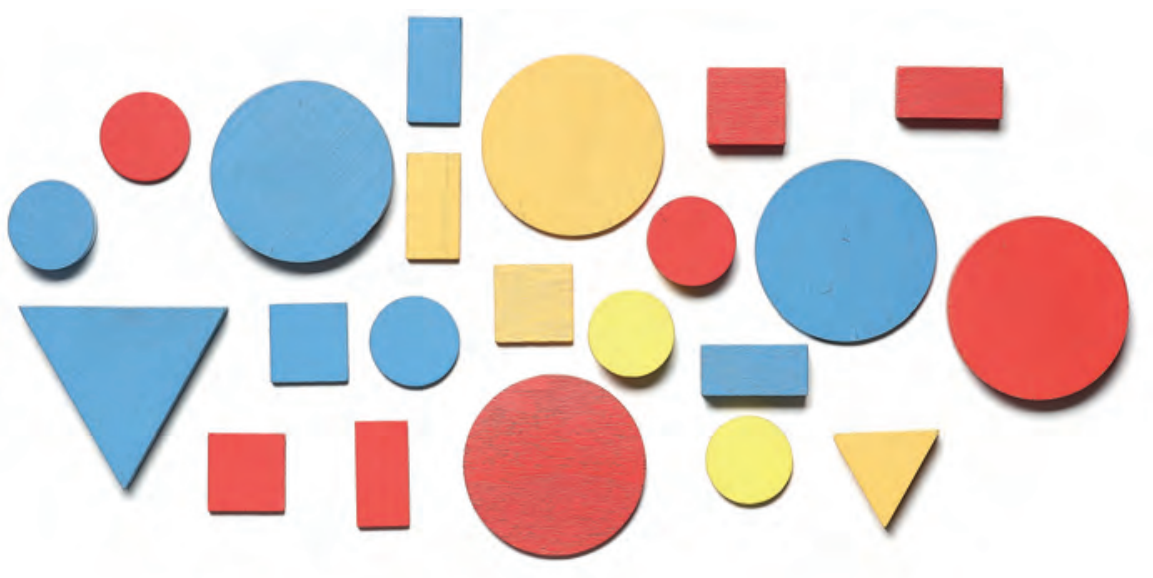

fentlich kolportierten, dass nämlich die mit den neuen Inhalten einhergehende Überforderung - für die es ihrerseits keine Belege gibt - Kinder krank mache. Wie ernst diese Aussagen genommen wurden, wird deutlich angesichts der Tatsache, dass die Frage vermeintlicher Gesundheitsschädigung es bis in die Parlamente schaffte und bei einer Anhörung in Baden-Württemberg 1974 ein eigens eingeladener Kinderpsychologe beschwichtigen mußte.

$[\ldots]$

Es verdient hier noch unbedingte Erwähnung, dass die Erwachsenen die Diskussion um die „Mengenlehre" unter sich ausmachten, während Grundschulkinder - und damit die Hauptabnehmer der Reform - nie systematisch zum Mathematikunterricht befragt wurden. Es finden sich jedoch diverse Quellen, die darauf hinweisen, dass die Kinder mitnichten überfordert waren, die neuen Inhalte viel schneller beherrschten als die Erwachsenen - mithin wohl auch häufig keiner Hausaufgabenhilfe bedurften - und generell Freude an ihrem Mathematikunterricht empfanden. Noch 1973 konstatierten 73 Prozent der betroffenen Eltern in Baden-Württemberg, der Unterricht mache ihren Kindern Spaß, während 18 Prozent angaben, ihre Kinder empfänden Widerwillen.

\section{Selbstbezügliches}

Ein Leser der scienceblogs hatte auf die Frage nach Erfahrungen mit der Mengenlehre noch eine offenkundig nicht ganz ernstgemeinte Antwort.

Jolly: Wenn ich mich richtig erinnere, wurde Mengenlehre an unserer Schule wieder abgeschafft, nachdem unter 3-Klässlern ein Streit ausbrach, ob es sowas wie eine Menge geben kann, die alle Mengen enthält, die sich nicht selbst enthalten, der von den Grundschullehrern nicht geschlichtet werden konnte.

Selbstreferentialität gibt es nicht nur in der Mengenlehre oder beim Beweis des Gödelschen Unvollständigkeitssatzes, sondern auch in Bielefeld. Dessen Stadtmarketing lobte im vergangenen August eine Million für den Beweis der Nichtexistenz ihrer Stadt aus, 5 was gewisse Fragen zur Selbstreferentialität aufwirft: Wenn bewiesen worden wäre, dass es Bielefeld gar nicht gibt, hätte die dann nichtexistierende Stadt trotzdem noch das Preisgeld auszahlen dürfen?

Soweit ist es jedoch nicht gekommen. Wie die Stadt im September mitteilte, ist keiner der rund 2000 Teilnehmer erfolgreich gewesen.

Unter den Beweisen waren auch ein paar harte Nüsse: Da wurde die Existenz Bielefelds mit Argumenten aus der Quantenphysik angezweifelt und komplizierte mathematische Formeln ließen eine ganze Großstadt auf dem Papier verschwinden. Aber wir blieben ruhig - und übersetzten mit Unterstützung von Wissenschaftlern der Universität Bielefeld und des Stadtarchivs Bielefeld auch das komplizierteste Fachchinesisch.

Zur Erinnerung an die Bielefeld-Verschwörung wurde ein 6oo Kilogramm schwerer Gedenkstein neben dem Leineweber an der Altstädter Nicolaikirche aufgestellt.

\section{Aufwändiges}

Als Nicolas Bourbaki mit seinen Lehrbüchern die reine Mathematik auf eine gemeinsame Grundlage stellen wollte, ging er nicht davon aus, dass Mathematik komplett formalisiert werden könne. Dementsprechend machte er sich keine Gedanken, welcher Ansatz mit Blick auf Formalisierbarkeit der effizienteste wäre. So erwähnte er in einem seiner Bücher, dass es hunderte, wenn nicht tausende Symbole benötige, wollte man seine Definition der Eins formalisieren.

Berühmt wurde das durch einen Text des Logikers A. R. D. Mathias aus den 8oer Jahren ${ }^{6}$ (veröffentlicht 1992 in The Mathematical Intelligencer). Der hatte seinen Kollegen Robert Solovay die genaue Anzahl der benötigten Symbole ausrechnen lassen, es waren 4523659424929. Und für die Berechnung von $1+1=2$ benötigt man bereits 19516572617436743593 Symbole.

Mathias ging es in seinem sehr leidenschaftlich geschriebenen Artikel nicht darum, die Unmöglichkeit einer 
vollständigen Formalisierung der Mathematik aufzuzeigen, sondern im Gegenteil Bourbaki für sein Desinteresse an Grundlagenfragen zu kritisieren, die ihn einen für die Formalisierung wenig geeigneten Ansatz hatte wählen lassen.

Bei Bourbaki war die Mengenlehre die Grundlage der Mathematik, einem von Hilbert vorgeschlagenen, vergleichsweise komplizierten Ansatz für die Logik erster Ordnung folgend. In einer späteren Ausgabe der Mengenlehre (1970) wählte Bourbaki einen anderen Zugang über „Kuratowski-Paare“, mit dem die formale Realisierung aber noch viel aufwändiger würde: die Definition der 1 benötigte nun 2409875496393137472149767527877436912 979508338752092897 Symbole.

Aktuell wieder in die Diskussion gekommen ist die Geschichte jetzt durch eine Frage von John Baez auf Mathoverflow 7 und die darauf erfolgte nochmalige Überprüfung mit einem Haskell-Programm von Alex Nelson:

For what it's worth, computing the size of 1 was nearly instantaneous, whereas computing the size of " $1+1=2$ " took about 7 minutes and 3 o seconds.

\section{Mengen oder Kategorien?}

Mengenlehre hat im 20. Jahrhundert - nicht erst durch Bourbaki - die Mathematik vereinheitlicht und auf eine gemeinsame Grundlage gestellt, sowie einen Rahmen für die Analyse von Fragen der Konsistenz und Beweisbarkeit geschaffen. Befürworter anderer Grundlagen argumentieren, dass beispielsweise die Kategorientheorie besser widerspiegele, wie Mathematiker tatsächlich denken, wie sie wirklich Mathematik betreiben oder welche mathematischen Strukturen wesentlich sind. (Dagegen argumentiert beispielsweise Timothy Chow in einer Diskussion auf Mathoverflow, ${ }^{8}$ dass die vorgeschlagenen alternativen Grundlagen vielleicht gut seien für die Homotopietheorie, aber nicht so geeignet für die numerische Lösung partieller Differentialgleichungen oder die Berechnung kleiner Ramsey-Zahlen. Andererseits könne man natürlich einfach annehmen, dass die gesamte Mathematik "sicher" ist und man bei Bedarf immer einen Weg fände, Methoden aus einem Zweig der Mathematik in einem anderen zu verwenden. Mit dieser Einstellung könne man sich dann einfach darum kümmern, Grundlagen für die Art von Mathematik zu finden, die einem wichtig ist; wenn meine Grundlagen für die Mathematik meinem Kollegen zu umständlich sind, dann ist das sein Problem und nicht meins.)

In einem im November im Sammelband Reflections on the Foundations of Mathematics ${ }^{9}$ veröffentlichten Artikel "What do we want a foundation to do? Comparing settheoretic, category-theoretic and univalent approaches" versucht Penelope Maddy die Suche nach „richtigen“ Grundlagen systematisch anzugehen, also zunächst zu fragen, was man von "Grundlagen der Mathematik“ erwarten sollte. Unter diesen Aspekten sollen die Theorien dann verglichen werden.
Die Mengenlehre bietet ein Risk Assessment für mathematische Theorien, eine Generous Arena, wo die Zweige der Mathematik in einem einheitlichem Rahmen und mit einem Shared Standard folgenden Beweisen ausgebaut werden können, und einen Meta-mathematical Corral, in dem formale Techniken auf die gesamte Mathematik auf einmal angewendet werden können.

$[\ldots]$

Was war also falsch an mengentheoretischen Grundlagen? Der erste Einwand ist, dass sich die Kategorientheorie mit unbegrenzten Kategorien befasst, wie der Kategorie aller Gruppen oder der Kategorie aller Kategorien oder der Kategorie aller mathematischen $X$, aber nichts dergleichen kann im Universum der Mengen gefunden werden. Grothendieck hat dieses Problem überwunden, indem er eine immer größere Folge von ,lokalen Universen' postuliert und feststellt, dass jede kategorientheoretische Aktivität in einem ausreichend großen dieser Universen ausgeführt werden kann. In mengentheoretischen Begriffen bedeutet dies, unerreichbare Kardinalzahlen hinzuzufügen, die kleinsten der großen Kardinalzahlen jenseits der ZFC-Mengenlehre. Mit anderen Worten, das Risiko von Grothendiecks Kategorientheorie ist nicht gröBer als das von ZFC + unerreichbaren Kardinalzahlen. Wenn Risk Assessment das grundlegende Ziel ist, ist die Mengenlehre immer noch in Ordnung. Ersatz für die Kategorien ist auch in der Generous Arena der Mengenlehre verfügbar, so dass Shared Standard und Metamathematic Corral ebenfalls in Ordnung zu sein sollten.

[...]

Obwohl im Universum der Mengen Ersatz für vieles zu finden ist, ist dieses Universum als Ganzes riesig, seine Konstruktionstechniken sind wild und wahllos, so dass es Horden nutzloser Strukturen enthält und - das ist der wichtige Punkt - keine Möglichkeit, die mathematisch vielversprechenden Strukturen unter den anderen auszuzeichnen. [...] Die Hoffnung bestand darin, Grundlagen zu finden, welche die Mathematiker zu den wichtigen Strukturen führen und diese strikt unter Bezugnahme auf ihre mathematisch wesentlichen Merkmale charakterisieren. Eine solche Grundlegung wäre tatsächlich nützlich für Mainstream-Mathematiker in ihrer täglichen Arbeit. Sie wäre nicht für diese weitgehend irrelevant wie die Mengenlehre; sie würde Essential Guidance liefern. Ihre Befürworter argumentierten, dass die Kategorientheorie genau dies für die algebraische Geometrie und die algebraische Topologie geleistet habe. [Meine Übersetzung, ebenso wie die folgenden Zitate.]

Maddy argumentiert dagegen, dass ja auch nicht gefordert würde, alle Mathematiker sollten wie Mengentheoretiker denken. Natürlich sollten algebraische Geometer oder algebraische Topologen nicht in mengen- statt kategorien- 
theoretischen Begriffen denken. Andererseits wäre es unvernünftig, wenn Analytiker oder Mengentheoretiker in kategorientheoretischen Begriffen denken sollten. Das habe durchaus auch Mac Lane so gesehen. Beide Grundlegungen hätten ganz unterschiedliche Ziele. Die Mengenlehre biete Risk Assessment, Generous Arena, Common Standard und Meta-mathematical Corral und erledige diese Aufgaben auch im Kontext kategorientheoretischer Grundlagen. Die Kategorientheorie biete Essential Guidance, aber nur für die Zweige der Mathematik mit einigermaßen algebraischem Charakter.

Das 21. Jahrhundert bringe nun neue Kritik an der Mengenlehre und einen neuen Kandidaten für ihre Rolle: „univalente Grundlagen“.

Das Programm der univalenten Grundlagen besteht darin, die Homotopietheorie zur Interpretation der Typentheorie von Martin-Löf zu verwenden und das sogenannte „Univalenzaxiom“ hinzuzufügen, welches grob gesagt den Effekt hat, isomorphe Strukturen zu identifizieren. Das Ergebnis soll „unvereinbar mit konventionellen Grundlagen“ (Awodey) und „eine völlig neue Grundlage“ der Mathematik (Voevodsky) sein.

Vladimir Voevodksy hat 2014 in einem sehr persönlichen Text $^{10}$ erzählt, wie ihn zahlreiche Fehler in eigenen und von ihm verwendeten Arbeiten motivierten, nach neuen Wegen zu suchen. Das habe ihn zu den univalenten Grundlagen geführt.

Dieses formale System ist keines, in dem irgendein Mathematiker tatsächlich etwas beweisen wollen würde. Unser Vertrauen in einen formalen Beweis beruht normalerweise auf unserem Vertrauen in den informellen Beweis, kombiniert mit unserer informierten Überzeugung, dass alle informellen Beweise formalisiert werden können. Dagegen sind die Anforderungen an die Beweisprüfung ganz andere: man braucht ein System, das formale Beweise darstellen kann, ein Werkzeug, das in der täglichen mathematischen Arbeit eingesetzt werden kann.

Maddy diskutiert verschiedene Beweisprüfungsverfahren und welche Rolle Homotopietypentheorie oder andere Grundlagen dort spielen können.

Zusammenfassend scheinen Risk Assessment, Metamathematical Corral, Generous Arena und Shared Standard weiterhin das Feld der mengentheoretischen Grundlagen zu sein. Es bleibt Proof Checking,

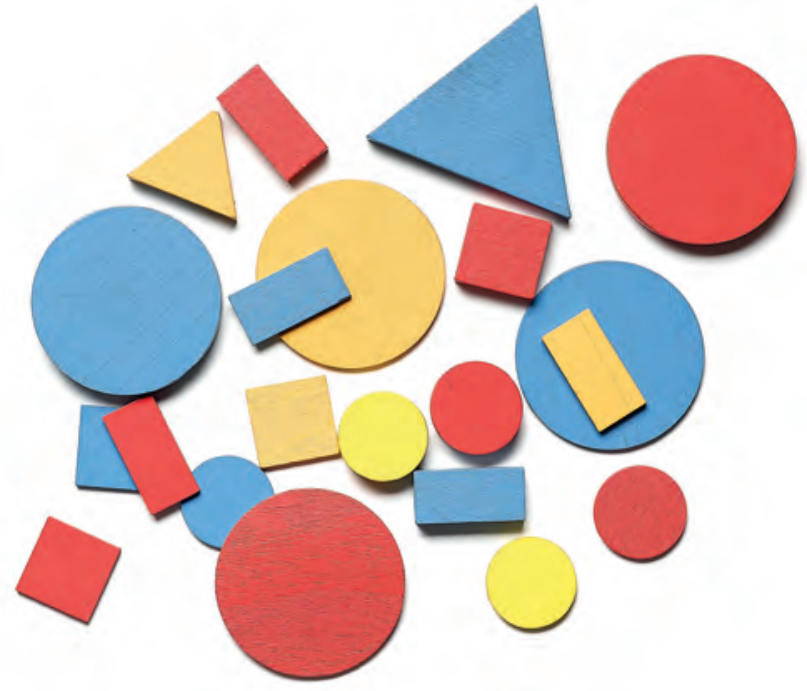

das neue Ziel, als Argument für univalente Grundlagen. Das Versprechen ist, dass gewöhnliches mathematisches Denken einfach und direkt in CIC ausgedrückt werden wird und die Gültigkeit von Beweisen dann automatisch in COQ überprüft werden kann, und dass die Homotopietypentheorie einen Rahmen für zuverlässiges Proof Checking bietet.

"[... I Ich weiß, wenn ich etwas getan habe, dann habe ich es getan und muss nicht später noch einmal darauf zurückkommen, noch muss ich mir Sorgen machen, ob meine Argumente zu kompliziert sind oder wie ich andere von ihrer Richtigkeit überzeugen kann. Ich kann einfach dem Computer vertrauen." (Voevodsky) Ich denke, wir können uns alle einig sein, dass dies ein sehr attraktives Bild ist, selbst wenn es sich nur auf Bereiche der Mathematik anwenden ließe, die für diese Art der Konzeptualisierung geeignet sind.

\section{Anmerkungen}

1. Die Zitate finden sich in zahlreichen Sprüchesammlungen im Internet und auch in verschiedenen Zeitungsartikeln. Den Ursprung oder Belege für die Echtheit der Zitate konnte ich aber nicht ausfindig machen.

2. www.spiegel.de/spiegel/print/d-41784469.html

3. tinyurl.com/y4njvzhw

4. tinyurl.com/y58ym6jj

5. www.bielefeldmillion.de

6. www.dpmms.cam.ac.uk/ ardm/bourbaki.pdf

7. tinyurl.com/y49ghtod

8. tinyurl.com/y5mvns4v

9. Doi $10.1007 / 978-3-030-15655-813$

10. www.ias.edu/ideas/2014/voevodsky-origins 